\title{
Design on Micro Blog Distribution System Based on Struts2 Framework
}

\author{
Zhe $\mathrm{Li}^{1, \mathrm{a}}$, Yuqiang Yang ${ }^{2, \mathrm{~b}}$, Houhua Shen ${ }^{3, \mathrm{c}}$ \\ ${ }^{1}$ College of International Exchange, Bohai University, Jinzhou, 121013, China \\ ${ }^{2}$ College of Information Science and Technology, Bohai University, Jinzhou, 121013, China \\ ${ }^{3}$ College of Engineering, Bohai University, Jinzhou, 121013, China \\ a503412508@qq.com, byyq8369@163.com, 'shenhouhuashh@126.com
}

\begin{abstract}
Keywords: Struts2 framework; micro blog distribution; system design; push and pull model; process design
\end{abstract}

\begin{abstract}
Micro blog content distribution becomes a bottleneck restricting the development of micro blog. In this paper, a study based on Struts2 framework provides technical support for the system development. Firstly, learning Struts2 framework, core controller, business controller and business logic components; then, designing push and pull model, a combination system of push and pull model is selected by comparing the Push model and Pull model, and the program process of pull model is designed; and finally, using a timing diagram to design micro blog release process, and having a detailed description for processing in server side. Results of this study solved the key technical problems of the system development, which play an important role in developing an affordable, powerful micro blog distribution system.
\end{abstract}

\section{Introduction}

Micro blog is a platform based on user relationship information sharing, dissemination and acquisition. Users set up individual communities through WEB, WAP and other clients, update information with 140 characters, and achieve instant sharing. Micro blog users can better express ideas and the latest developments at all times, and blog is more emphasis on combing their sees, hears, and feels over a period of time. Micro blog platform has the following characteristics [1,2]: first, convenience, publish information quickly and information dissemination quickly. As a viewer, you can browse information of interest in the micro blog; also as a publisher, you can publish content on the micro blog for others to browse. Second, originality, civilians and a high level of writers are pulled to the same level because of 140-character limit, leading to a large number of original content to be produced. Third, grassroots, micro blog users have a strong grassroots, and they are widely distributed on multiple platforms, such as desktop, browser and mobile terminals, a variety of business models coexist. With micro blog has been accepted by more and more people, micro blog content distribution becomes a bottleneck restricting the development of micro blog. In this paper, a study based on Struts2 framework provides technical support for the system development.

\section{Struts2 Framework Structure}

Struts is the most popular Web framework currently, it is very simple and easy to develop MVC program by using it. Struts2 is the next generation of product of Struts, which is a new MVC framework merged by struts and WebWork. There is a huge difference between the new Struts2 architecture and Struts1 architecture. Struts2 is WebWork-centric, using mechanism of interceptor to handle the user's request, this design also completely separates business logic controller from Servlet API, so Struts2 can be understood as updated product of WebWork [3,4].

Struts2 framework can be divided into three parts: the core controller FilterDispatcher, business controller Action and business logic components. Among them, the core controller FilterDispatcher is the basis for Struts2 framework, including the control process and processing mechanism inside the frame. Business controller Action and business logic components require user to implement. 
While users developing Action and business logic components, they also need to write the relevant configuration file for the core controller FilterDispatcher to use; business controller Action is wrote and implemented by developers, Action class can be a simple Java class, which is completely separated from Servlet API. Action generally has a execute() method, you can also define other business control methods; the business model components can be a model to implement business logic, even EJB, POJO or JavaBean, in the actual development, the distinction and definition of business model components is relatively vague, it is also beyond the scope of Struts2 framework. Different developers or teams have their own ways to achieve business logic models, and the purpose of Struts 2 framework is to use Action to invoke business logic models. Struts2 framework structure is shown in Fig. 1.

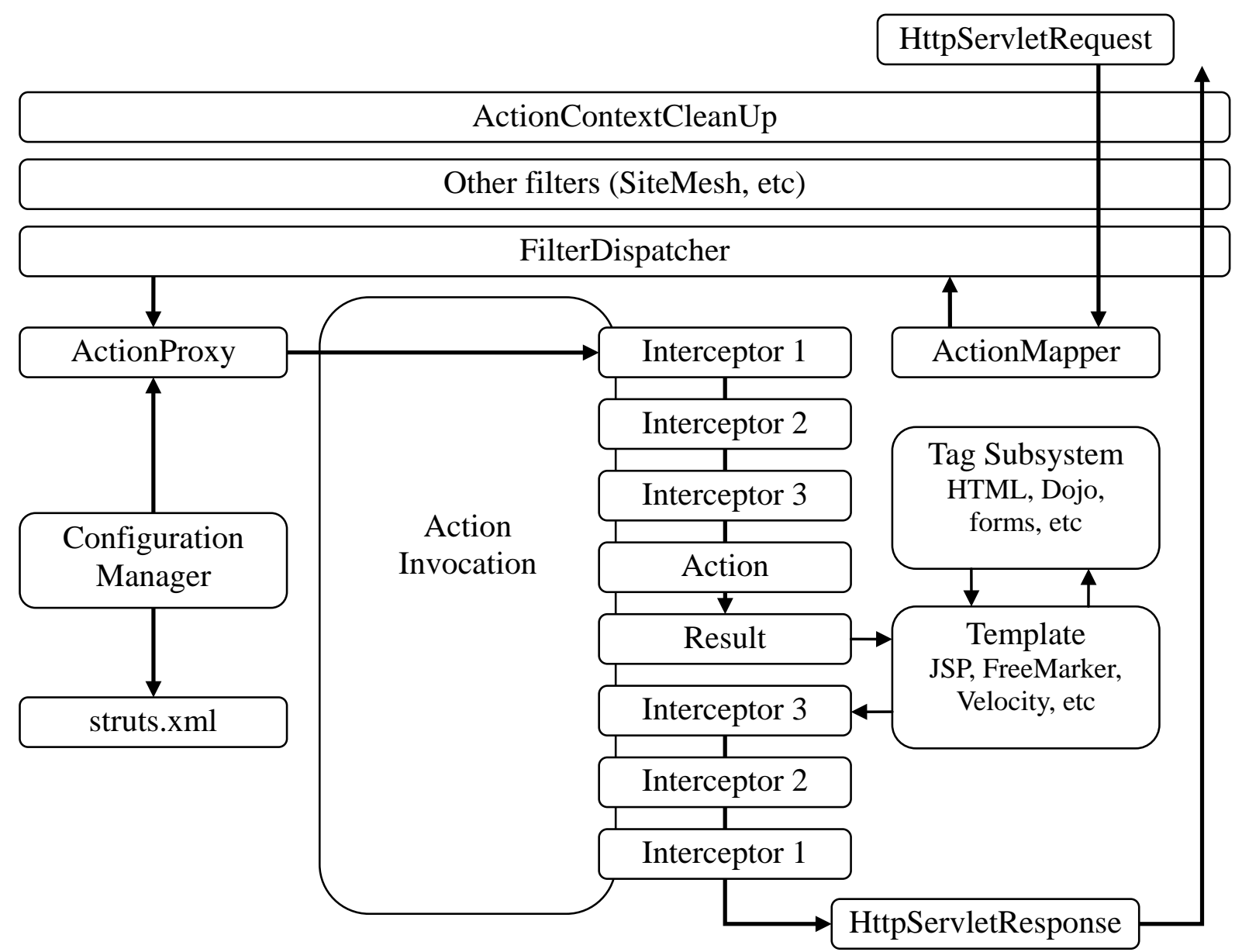

Fig. 1. Struts2 Framework Structure

\section{Push and Pull Model Design}

In the traditional Client/Server architecture, information acquisition way is based on "Pull" model, the server handles in accordance with the service request sent by the user terminal, and then returns desired results. In the Push system, the server needs to "Push" information to the user terminal system. Both the direction of data transmission is from the server to user, but the initiator of the operation is different. From the relationship between "Source" and "user", the flow of information can be divided into two models, namely, they are information push model and information pull model. Comparison of the two models is shown in Table $1[5,6]$.

Push and pull model is the core of the micro blog content distribution system. This system combines the advantages of push and pull to design a combination system. Push model is to push for the on-line users, when users send micro blog, the system will push the number of new micro blog to user' fans, after users see the prompt of new micro blog, click on the specific figures will trigger the system to pull new micro blog content. Push pressure will disperse to online users, and 
client and server of them maintain a long link, so the system uses the server to push to the user client. It combines the advantages of push model to pull model, not only send micro blog fast, but also the pressure of pull micro blog can disperse to each user. The underlying implementation of specific pull model is achieved by pull model file system. Program flow of single user pulls micro blog list is shown in Fig. 2.

Table 1. Comparison between Push mode and Pull mode

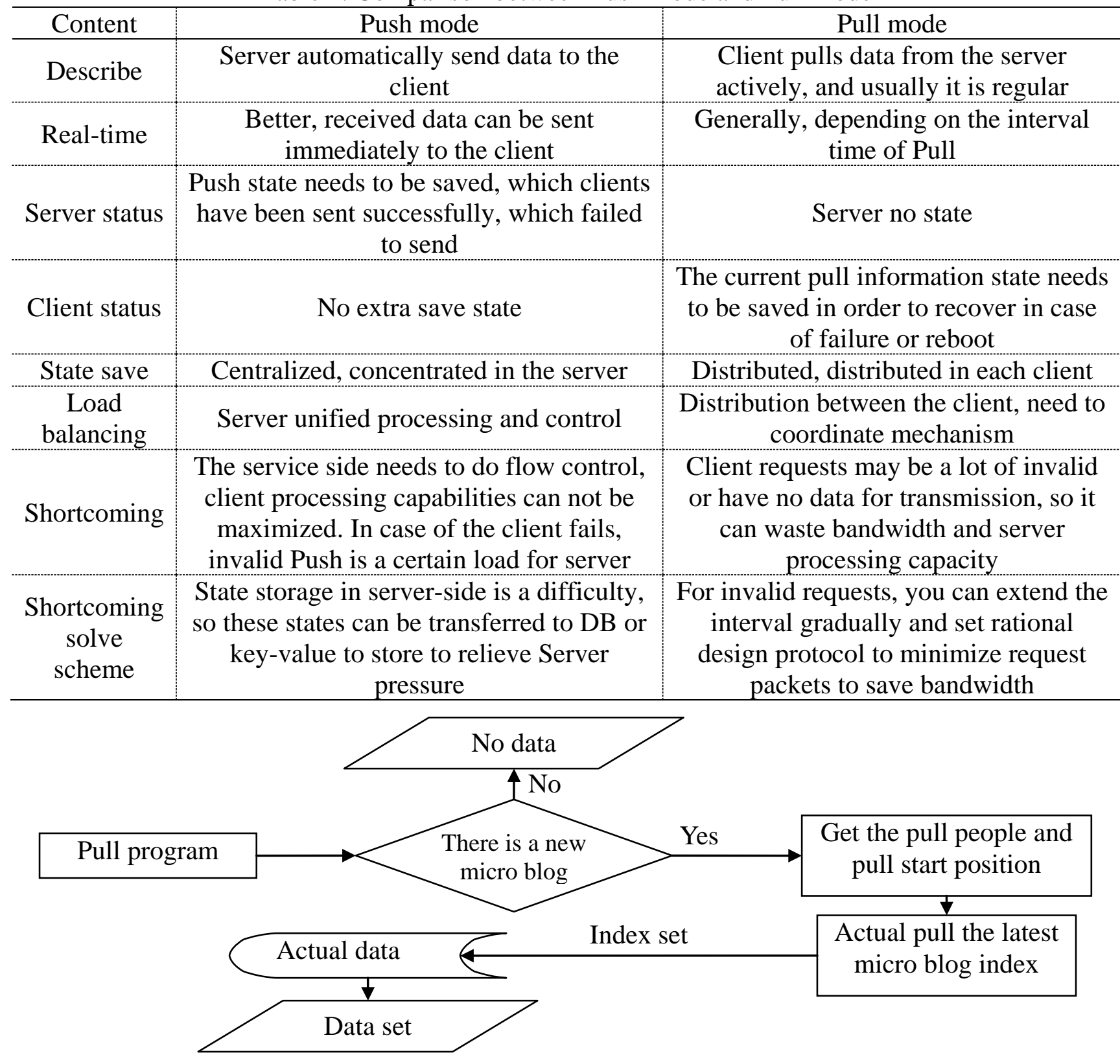

Fig. 2. Program flow on "Pull" mode

After logging in for the first time, pull program is triggered when pull micro blog list. Pull program requires userid attribute as an index, and first locate the user's directory files, use the user's userInfo file to determine whether there is a new micro blog. The last read position as a starting position when you read lastRead field, then obtain needed pull personnel, judge that you need to find new micro blog in those groups through personnel, the latest data is written in userThing of personal directory, userThing file can store up to 500 micro blog index records, and then pull the corresponding numbers to return to index collection, then access specific data through the index. Many of these specific data is to use memcache to store in memory, so it can return data quickly, then return to the front-end through an API layer processing data format. Wherein, the pull specific data also need to determine the appropriate circle information. 


\section{Process Design on Micro Blog Release}

Micro blog types that users can publish include text, forwarding, picture, document and sharing link [7]. Because different micro-blog types of users, the system must provide the tag variables to distinguish different types of micro blog. The tag variable is assigned by the client-side JavaScript codes, and is submitted to the server by the AJAX with the main content of micro blog together. When publish a new micro blog in the client browser, the browser sends a request asynchronously. After the server receives the request, it generates the micro blog instance storing in the database. Micro blog data is persisted, the system will generate a JSP page code based on received micro blog information, and this code will be returned to the client browser.

Sequence diagram shows the interaction between objects, these objects are arranged in chronological order. Timing diagram of micro blog release is shown in Fig. 3.

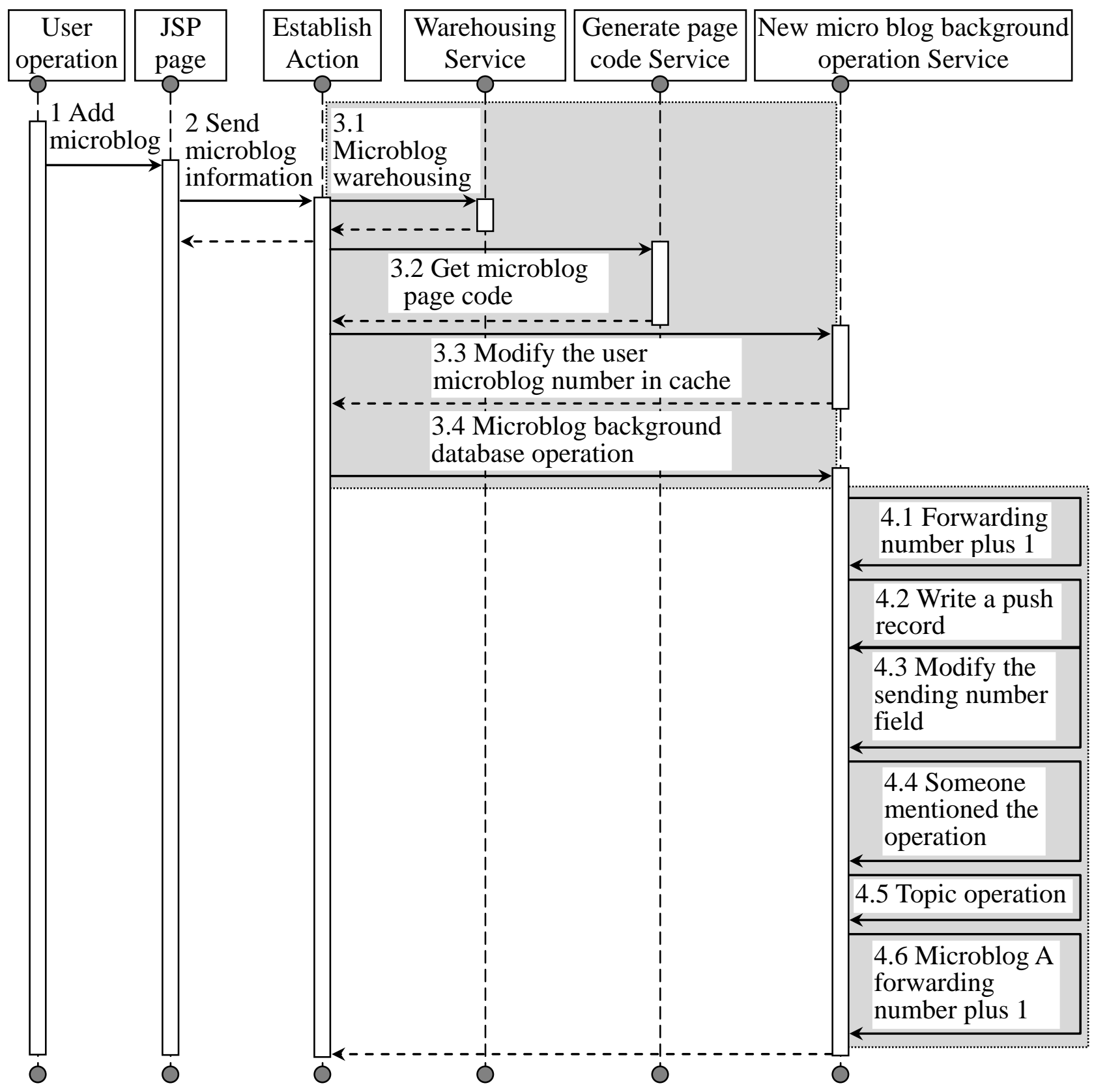

Fig. 3. Sequence diagram on micro blog release

In Fig. 3, the processing of background for the user data and micro blog data is described as follows:

(4.1) Number of forwarding adds one. If forwarded, the number of forwarding for that micro blog will add one; 
(4.2) Write push record. Obtaining the user's fan list, writing push record to the table of feeds;

(4.3) Modify the number of have been released fields. Modifying the number of that have been released micro blog fields for the user information table in database;

(4.4) Referring to a person operation. Operating "someone mentioned" in micro blog to operate database tables;

(4.5) Topic operation. Operating topic in micro blog, updating the database tables;

(4.6) Forwarding number of micro blog $A$ adds one. If forwarded micro blog A contains that "micro blog B is forwarded by A ", then step 2 operates on micro blog B, and therefore forwarding number of micro blog A needs to be added one in Step 7.

\section{Conclusion}

Struts2 is the most widely used MVC framework currently in Java field, has the following advantages [8]: clear hierarchy, so that programmers can only focus on business logic implementation; rich tag library, the efficiency of development is greatly improved; rich interceptor implementation; through the configuration file, you can grasp the relationship between the various parts of the whole system; exception handling mechanism, simply configure exception mapping in the configuration file, you can do the appropriate treatment for exception; highly extensibility, core jar package consists a struts-default.xml file, default type of bean and resultType and interceptor stack are set in the file, all of these default settings, users can take advantage of configuration file to change to achieve pluggable characteristic of framework for plug; aspect-oriented programming ideas, the interceptor is a small functional unit, users can merge them into one large interceptor. Based on the paper design, if these advantages are fully exploited in the process of software development, an affordable, powerful micro blog distribution system can be developed.

\section{Acknowledgement}

This work is supported by social science fund project of Liaoning province (L13BGL013): Research on management practice and development strategy of government microblogging in Liaoning province.

\section{References}

[1] P. X .Lin, "Research and Development of Microblog Information Retrieval System," Master's degree of Central China Normal University, 2014.

[2] Baidu encyclopedia, "Microblog," http://baike.baidu.com/link?url=bGAwBwXg_AC, 2016-1-8.

[3] Y. F. Zou, H. W. Luo, "Implementation of Defeat Management System Based on Frame of Struts+Spring+Hibernate," Computer Technology and Development, vol. 22, no. 2, pp. 146-148, 2012.

[4] Y. J. Liu, J. C. Kang, W. F. Lu, "Overview of Model-Driven Architecture," Computer Science, vol. 33, no. 3, pp. 226-228, 2006.

[5] Pi9nc column, "The Push/Pull mode analysis of message system," http://cache.baiducontent.com/c?m=9f65c \%BD\&qid=889d84940002a2ce\&p1=2, 2016-1-8.

[6] R. Y. Li, "Distributed Real-time Delivering MicrobIogging System," Master's degree of South China University of Technology;, 2011.

[7] M. Wang, X. Jing, S. Q. Li, et al, "Based on Hbase agricultural microblog data storage and processing strategy," Journal of Chinese Agricultural Mechanization, vol. 35, no. 3, pp. 221-225, 2014.

[8] X. B. Chen, "Advantages of struts2," Dhttp://xiaobing259-163-com.iteye.com/blog/1530960, 2016-1-8. 